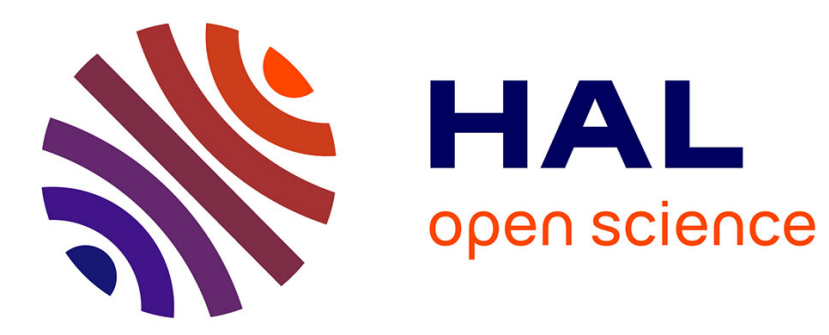

\title{
Observer et représenter des usages
}

Bernard Yannou, François Cluzel

\section{To cite this version:}

Bernard Yannou, François Cluzel. Observer et représenter des usages. Déployer l'innovation: Méthodes, outils, pilotage et cas d'étude, Techniques de l'Ingénieur, 2015, 978-2-85059-129-7. hal-01683491

\section{HAL Id: hal-01683491 \\ https://hal.science/hal-01683491}

Submitted on 13 Jan 2018

HAL is a multi-disciplinary open access archive for the deposit and dissemination of scientific research documents, whether they are published or not. The documents may come from teaching and research institutions in France or abroad, or from public or private research centers.
L'archive ouverte pluridisciplinaire HAL, est destinée au dépôt et à la diffusion de documents scientifiques de niveau recherche, publiés ou non, émanant des établissements d'enseignement et de recherche français ou étrangers, des laboratoires publics ou privés. 


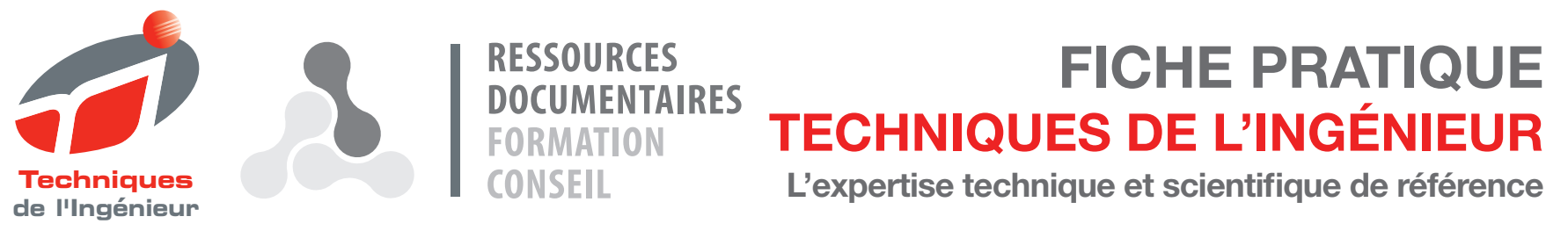

\title{
1448 \\ Observer et représenter des usages
}

\author{
Bernard YANNOU \\ Professeur à l'École Centrale Paris, directeur adjoint du laboratoire Génie Industriel \\ François CLUZEL \\ Enseignant-chercheur, Laboratoire Génie Industriel,École Centrale Paris
}

Cette fiche pratique fait partie de la base documentaire :

Déployer l'innovation

Dans le pack :

et dans l'univers : Génie industriel

Cette fiche peut être traduite dans la langue de votre choix.

Accédez au service Traduction à la demande dans votre espace « Mon compte ». (Service sur devis)

Document délivré le : 30/09/2015

Pour le compte : 7200049198 - ecole centrale de paris // 138.195.100.152

Pour toute question :

Service Relation clientèle - Techniques de l'Ingénieur

249 rue de Crimée - 75019 - Paris

par mail infos.clients@teching.com ou au téléphone 0033 (0) 153352020 


\title{
Observer et représenter des usages
}

\author{
par Bernard YANNOU \\ Professeur à l'École Centrale Paris, directeur adjoint du laboratoire Génie Industriel \\ François CLUZEL \\ Enseignant-chercheur, Laboratoire Génie Industriel, École Centrale Paris
}

Vous avez commencé à structurer un projet d'innovation à partir d'une idée initiale. La connaissance des usagers, ou plus largement des bénéficiaires du produit ou service que vous souhaitez développer, est absolument essentielle. Pourtant peu de porteurs de projet aujourd'hui prennent réellement le temps d'observer.

Cette fiche vous permettra de comprendre l'importance de l'observation, mais également de la représentation des usages en définissant un protocole en adéquation avec vos objectifs. Vous serez ensuite à même d'analyser les résultats et de choisir un mode de représentation adapté pour formaliser et capitaliser votre expérience, mais surtout pour mettre à jour les problèmes et les douleurs des usagers et ainsi convaincre les décideurs de la justesse de votre raisonnement.

L'observation et la représentation des usages est le troisième livrable de la méthodologie d'innovation radicale tirée par les usages, Radical Innovation Design ${ }^{\circ}(\mathrm{RID})$, décrite en détail au travers de différentes fiches pratiques. Après avoir structuré le projet et défini le besoin idéal, ce livrable permet de rentrer véritablement dans les usages, problèmes et douleurs des utilisateurs ou bénéficiaires, liés aux produits et pratiques existantes. II permet d'initier une analyse poussée des usages et des problèmes afin de mettre à jour des poches de valeurs, combinaison significative d'un problème et d'un usage non ou mal couverte par les solutions existantes.

\section{En pratique}

\section{Étape 1}

\section{Pourquoi observer et représenter des usages ?}

L'observation et la représentation des usages permettent réellement d'entrer dans le processus d'identification et d'analyse des problèmes et usages existants, processus essentiel pour déceler les lieux d'innovation, également appelés poches de valeur dans la méthodologie Radical Innovation Design ${ }^{\circledR}$. Une poche de valeur est la combinaison d'un usage et d'un problème non ou mal couvert par les solutions existantes et dont l'intérêt est justifié par l'importance du problème (par exemple, le problème est-il fréquent et ses conséquences désastreuses ?) ou la taille de l'usage (par exemple, la population concernée est-elle importante ?). Afin de développer des innovations radicales tirées par les usages et l'utilité créée (réduction de « douleurs" ou problèmes), l'observation physique est primordiale dans ces situations d'usage problématiques.

Pour être plus précis, l'observation vous permettra de :

- comprendre des usages: les contextes des situations ;

- comprendre la situation et le comportement des utilisateurs : qui sont-ils, leurs motivations, les problèmes qu'ils rencontrent, parfois sans même s'en rendre compte ;

- comprendre les interactions avec d'autres objets de la situation d'usage ;

- mesurer tous ces aspects : fréquences, probabilités, caractérisation des problèmes et de leurs conséquences. Les mesures et leur mise en œuvre vont s'avérer primordiales dans RID.

L'observation est définie soit comme une activité permettant de recevoir de l'information du monde extérieur au travers de nos sens, soit comme l'enregistrement de données en utilisant des instruments scientifiques. La notion d'observation présentée dans cette fiche se situe à la croisée de ces deux définitions. Vous allez observer, c'est-à-dire monter en compétences et connaissances sur des situations d'usage, des problèmes, sur vos usagers, de manière scientifique et structurée, en capitalisant un savoir pour le réutiliser de manière opportune dans la suite de votre projet d'innovation.

L'usage, quant à lui, se définit comme une activité intentionnelle : on veut se servir ou employer quelque chose pour répondre à un besoin. L'usage est directement lié à la notion de fonction. II peut également être défini comme une pratique ou une coutume observée dans un groupe, dans une société (dictionnaire Larousse). L'usage, dans une optique de développement de nouveaux produits, peut ainsi être vu comme une mise en situation où un usager avec une intentionnalité vit une histoire où il entre en interactions avec des éléments d'un décor et d'autres personnages. L'observation des situations d'usage consistera alors à capter et analyser ces interactions.

Dans la méthodologie RID, les usages ne doivent pas, dans la mesure du possible, mettre en scène une solution de produit ou service qui résout partiellement les problèmes mis en scène. Ils doivent décrire les usages existant en dehors de toute solution spécifique ; ces solutions apparaîtront plus tard dans le processus RID sous la forme de "solutions existantes ". Bien sûr, il reste parfois difficile de s'abstraire de certains produits ou services « basiques » entrés depuis longtemps dans le quotidien des utilisateurs. 


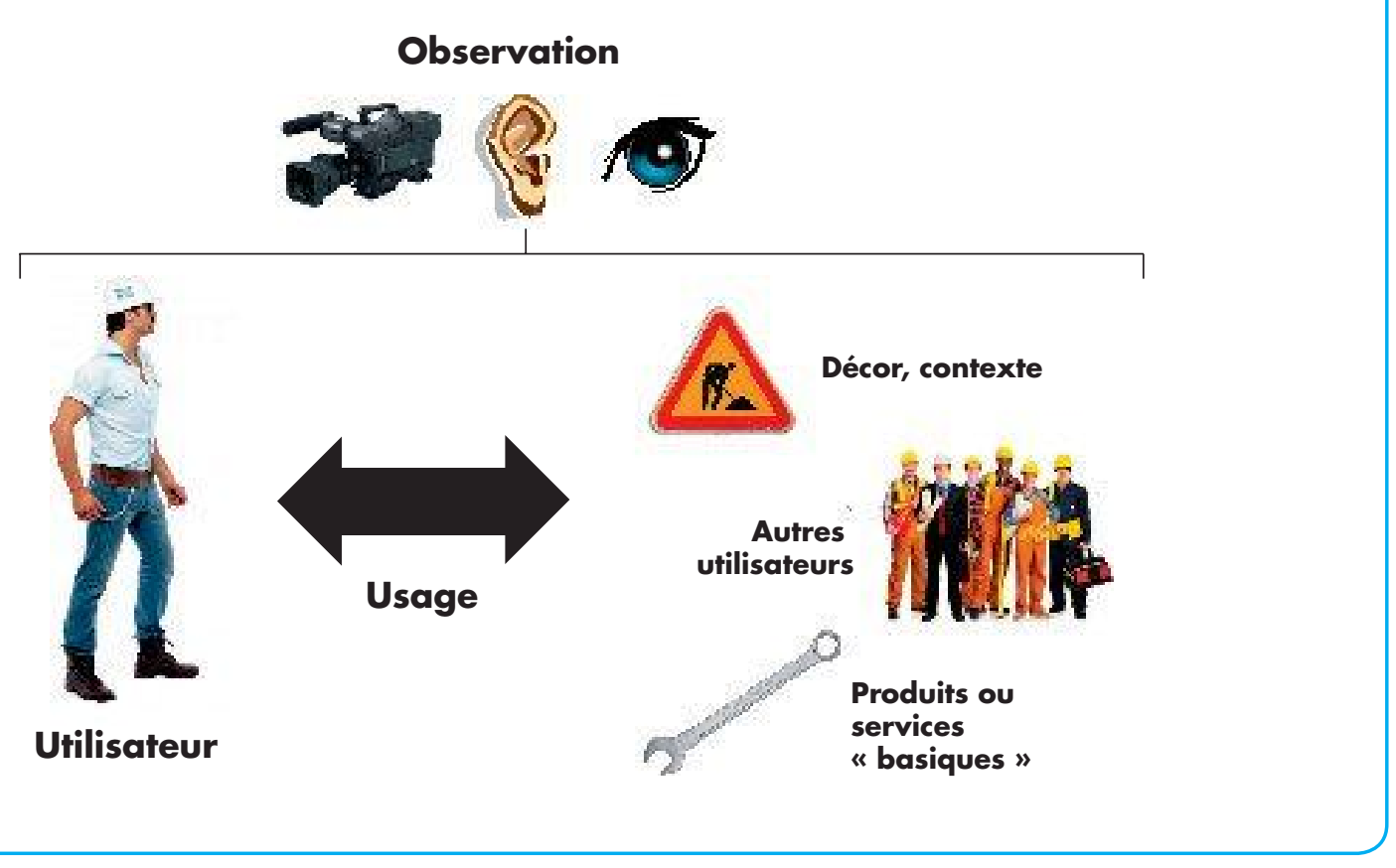

De nombreuses techniques d'observation peuvent être mobilisées, tout comme de nombreux moyens techniques d'observation peuvent être utilisés. Cette fiche vous guidera à définir le bon protocole, analyser les données et représenter de manière pertinente les usages pour servir votre projet d'innovation.

\section{Étape 2}

\section{Définissez un protocole d'observation}

Définir un protocole d'observation passe forcément par la définition des objectifs de l'observation. Par cette observation, que voulez-vous mesurer, quantifier, atteindre, et pourquoi ? Dans quel contexte souhaitez-vous réaliser cette observation ? Qui pensez-vous observer, et faisant quoi ? En connaissant précisément ces éléments, vous serez ainsi en mesure de définir un ensemble de variables d'observation, qu'elles soient qualitatives ou quantitatives.

Plus généralement, vous allez observer un objet ou une série d'objets (au sens large), manipulé par un utilisateur qui a un certain comportement, le tout dans un environnement où se trouvent probablement d'autres objets et d'autres personnages. Pour dimensionner précisément votre observation, vous devez vous intéresser en détail à :

- l'intention de l'usager ou la logique de la situation d'usage,

- l'activité que vous observez ;

- la fonction du ou des objets observés ou du décor mis en scène ;

- le système au sein duquel votre observation s'insère.

Une fois les variables définies, vous et votre groupe projet êtes désormais en mesure d'imaginer au cours d'une séance de créativité un ensemble d'expérimentations, qui vous permettront de mesurer ces variables et d'observer les phénomènes désirés. Pour chaque expérimentation, définissez précisément :
- le contexte d'observation :

- les acteurs impliqués (utilisateurs ou autres) ;

- le matériel nécessaire ;

- les livrables ;

- le mode de représentation, qui sera décrit dans la suite de cette fiche.

N'hésitez pas à tester votre protocole dans différents cas de figure avant de vous lancer sur le terrain. Une fois l'observation lancée, capitalisez un maximum d'informations. Mieux vaut avoir trop de données que pas assez!

De nombreuses techniques d'observation existent. Choisissez celle qui est la plus adaptée à vos objectifs. Vous pouvez pour cela consulter l'excellent site web (en anglais) "Designing with people », dont l'adresse vous est fournie à la fin de cette fiche. Les techniques proposées vont de la simple interview ou du simple questionnaire à des techniques plus évoluées comme le "Day in the life » (qui consiste à observer et enregistrer une journée de la vie d'un utilisateur) ou la technique des personas. Un persona est un personnage imaginaire, basé sur des personnes réelles, et représentant un archétype d'utilisateurs, donc très représentatif. II permet d'illustrer le comportement d'une catégorie d'utilisateur. Attention cependant, un persona au sens de Radical Innovation Design $₫$ doit être très précisément défini ; vous devez au maximum quantifier et qualifier la population à laquelle il se rattache et justifier en quoi ce persona est qualitativement et surtout quantitativement représentatif des usagers visés. Retenez que l'utilité d'un nouveau produit ou service, qui est un élément déterminant du succès du lancement d'une innovation, dépend de la taille de la population pour laquelle on sera capable d'apporter une valeur ajoutée dans des situations d'usage pour lesquelles les problèmes auront en partie au moins été résorbés.

Le modèle de fiche « Usage » - Réf. Internet : dtou6473 vous propose un modèle de document utilisable pour 
définir un protocole d'observation et synthétiser les résultats qui en découlent. L'analyse de ces résultats est présentée à l'étape suivante. L'exemple de fiche "Usage » : le plaquiste - Réf. Internet : dtou6474 propose un exemple d'application de ce modèle de document sur l'exemple du métier de plaquiste.

\section{Étape 3}

\section{Analysez les résultats de l'observation}

Une fois les protocoles d'observation mis en œuvre et les données récoltées, un gros travail d'analyse des résultats de l'observation vous attend. Nous n'allons pas ici rentrer dans les méthodes d'analyse, qui peuvent être extrêmement diverses, de la plus qualitative à la plus quantitative, en fonction du type d'observation mené.

Cependant, l'objectif de ces analyses sera de mettre à jour les problèmes, les douleurs, les dysfonctionnements associés aux situations d'usage observées. Les produits existants sont-ils satisfaisants ? Si aucun produit existant n'est utilisé, les pratiques existantes sont-elles satisfaisantes? De nouveaux besoins peuvent-ils être identifiés, quantifiés ?

Par ailleurs, les protocoles développés et les observations menées ont-ils eux-mêmes été satisfaisants ? Les premiers résultats permettent-ils de lancer de nouvelles observations plus ciblées ou mieux structurées ?

Afin d'illustrer de manière très simple une observation et l'analyse qui en découle, vous trouverez ci-dessous un exemple concret.

\section{Exemple : le fauteuil de handitennis (observation d'un usage)}

Dans le cadre d'un projet d'innovation Radical Innovation Design $®$ à l'Ecole Centrale Paris, des étudiants ont travaillé sur le fauteuil de handitennis d'une de leur camarade, joueuse de haut niveau qui souhaite remporter les jeux paralympiques de Rio en 2016. Son idée initiale était d'alléger son fauteuil pour gagner en vitesse de déplacement et diminuer sa fatigue, mais le besoin idéal de ce fauteuil a été défini comme devant " permettre à la joueuse de gagner tous les points d'un match de handitennis dans les situations de jeu représentatives ». C'est bien sûr un besoin idéal, qui ne sera probablement jamais atteint, mais toute solution innovante devrait chercher à s'en approcher au maximum.

À partir de ce besoin, les étudiants ont défini plusieurs protocoles d'observation pour bien comprendre les différentes situations d'usage (ici de jeu) du fauteuil et les problèmes rencontrés par son utilisatrice. Par exemple, les étudiants l'ont observée au service. Ils ont cherché à qualifier et quantifier les mouvements du fauteuil pendant un service, la rotation des roues, la performance de la joueuse (pourcentage de bons services) et la vitesse de la balle. Ils ont pour cela simplement demandé à la joueuse de servir un certain nombre de fois et l'ont filmée et photographiée. Assez rapidement, il est ressorti qu'il existe un mouvement de «twist » du fauteuil, et un mouvement de recul. Ces mouvements limitent la puissance de la balle en "consommant » de la puissance de la joueuse lors du service. Les étudiants ont alors continué l'observation en bloquant la rotation d'une, puis des deux petites roues situées à l'avant du fauteuil, qui servent principalement à orienter le fauteuil (voir photos).
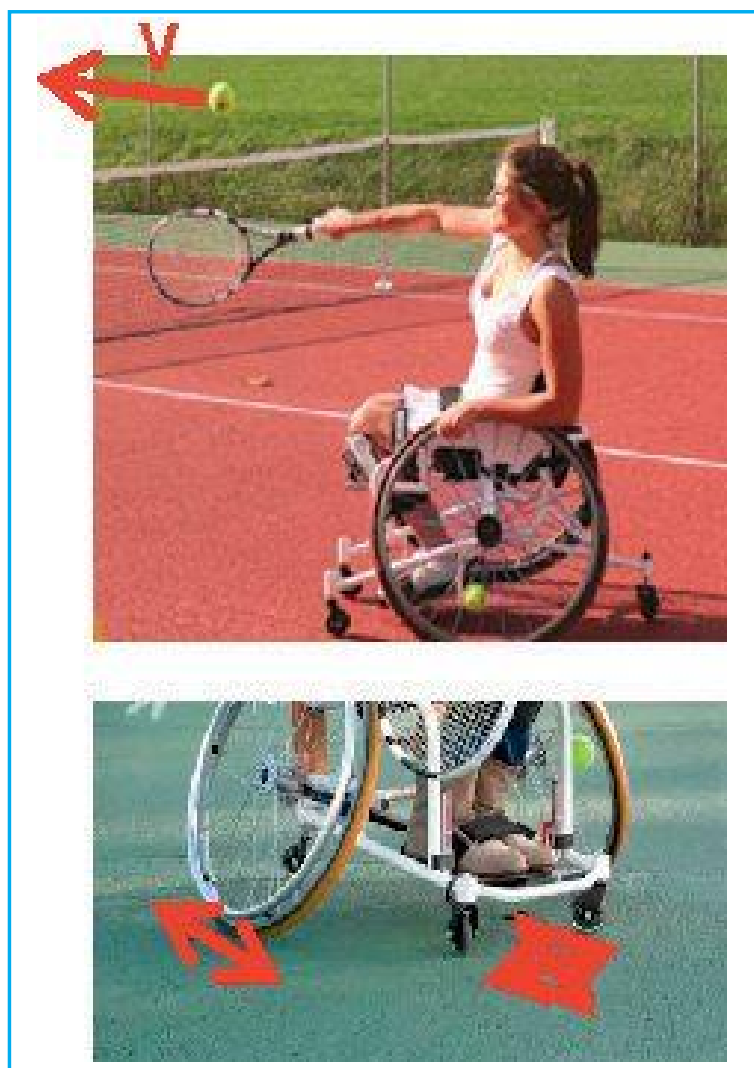

Avec l'accord de la joueuse de tennis

Le résultat de l'observation a été le suivant : en bloquant une des deux roues, la joueuse a gagné $9 \%$ de vitesse au service, et $19 \%$ en bloquant les deux roues ! Pour autant elle a perdu en précision car elle n'était pas habituée à cette nouvelle configuration, précision qu'elle a rapidement retrouvée au bout de quelques heures de pratique.

Ce résultat est d'autant plus important que d'autres tâches d'investigation menées auprès d'entraineurs de handitennis ont montré que $80 \%$ des services d'un match sont gagnants !

Les étudiants ont alors poursuivi leur projet en poursuivant la méthodologie Radical Innovation Design $®$ pour développer une solution permettant de bloquer les roues le temps du service, en accord avec les règles de la fédération internationale. On est bien loin de l'allégement du fauteuil initialement envisagé.

\section{Étape 4}

\section{Représentez les usages}

Vous avez finalisé vos observations et analysé les problèmes associés aux situations d'usage. Afin de communiquer ces informations (à votre groupe projet, aux décideurs, au public...) et convaincre vos interlocuteurs, il vous faut maintenant représenter de manière synthétique et efficace ces scénarios d'usage existants. La méthodologie Radical Innovation Design $®$ propose de le faire de manière très imagée. C'est en effet un excellent moyen pour communiquer, et donc convaincre. Pour autant, l'aspect ludique de la représentation ne doit pas prendre le dessus sur la rationalité de votre approche d'innovation et au détriment des informations qui doivent s'y raccrocher. 
Ainsi, une bonne représentation de scénario d'usage :

- est une histoire, avec un début et une fin ;

- met en jeu des usagers dans des situations d'usage archétypales représentatives d'une réalité ;

- introduit et met l'accent sur des problèmes rencontrés (dans l'exemple du handitennis, mouvements inattendus du fauteuil et perte de puissance)

- doit être quantifié : combien de personnes sont-elles concernées, quelles sont les observations (dans l'exemple du handitennis : vitesse de balle, nombre d'aces), quelle est la probabilité, la fréquence et l'importance des problèmes ?) ;

- doit être crédible et même validée statistiquement par les observations ou d'autres données d'étude.

Nous proposons ci-dessous plusieurs techniques de représentation d'un scénario d'usage.

Texte

Un scénario d'usage peut simplement être décrit par un texte, racontant une histoire. C'est bien sûr la technique la plus rapide et la plus économe en ressource, mais qui peut s'avérer trop simpliste pour permettre à votre interlocuteur d'imaginer ce que vous souhaitez montrer.

\section{Story-board/bande dessinée}

Cette technique empruntée au cinéma permet de représenter une histoire sous forme d'un enchaînement de dessins accompagnés de paroles ou de légendes. C'est également une technique très rapide (peu importe la qualité artistique de votre œuvre !), très utile lorsque vous n'avez pas pu photographier ou filmer de vrais utilisateurs. Quelques ressources en ligne, listées dans la rubrique FAQ de cette fiche, permettent de réaliser simplement de telles représentations.

Roman photo

Le roman-photo est très semblable à la technique précédente, à la différence que ce sont des photos qui sont manipulées. Les moyens nécessaires sont encore une fois très limités. Les photos peuvent représenter soit de vrais utilisateurs (cf. exemple du handitennis), soit, par exemple, des Legos $®$ ou des Playmobils $₫$ qui vous permettront de représenter ce que vous n'avez pu photographier.

\section{Exemple : le fauteuil de handitennis (représentation d'un scénario d'usage)}

Les étudiants travaillant sur le fauteuil handitennis ont représenté quelques scénarios d'usage sous forme de roman-photo. Un exemple est reproduit ci-dessous.

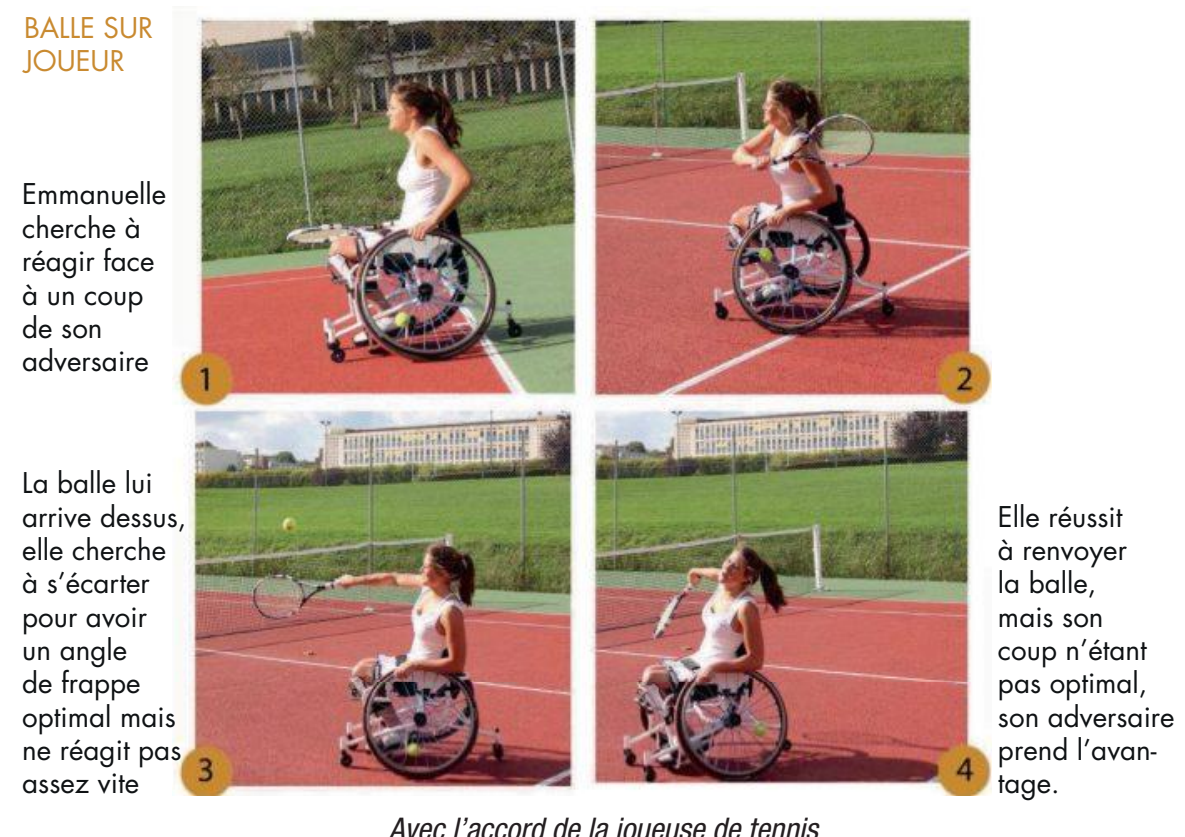

Cette représentation est claire et efficace, mais une représentation sous forme de film aurait peut-être été préférable puisqu'elle montre la dynamique des mouvements.

\section{Film}

Le film est un moyen évident de représentation d'un scénario d'usage. II est en revanche un peu plus compliqué à mettre en œuvre que les techniques précédemment citées, car il peut nécessiter un montage et un travail de post-traitement assez long. Vous pouvez soit filmer des situations vécues par de vrais utilisateurs, soit vous filmer vous-mêmes et les membres de votre équipe projet pour « mimer » les situations-types et donc devenir acteurs.

Film d'animation

Le film d'animation mérite d'être cité car il peut se révéler très utile lorsque vous n'êtes pas en mesure de filmer des utilisateurs réels mais que la technique reste adaptée à vos objectifs. Quelques ressources en ligne, listées dans la rubrique FAQ de cette 
fiche, permettent de réaliser simplement des films d'animation.

\section{Film d'animation en volume}

Un film d'animation en volume, également appelé animation image par image (stop motion en anglais) est une technique permettant de créer une animation à partir d'images assemblées les unes à la suite des autres. Elle se situe donc entre le story-board et le film d'animation. Elle est particulièrement adaptée si vous souhaitez une représentation dynamique à base de Lego $₫$ ou Playmobil@. Quelques films de ce type sont accessibles depuis la rubrique Sites internet de cette fiche.

\section{Démonstration}

Une dernière technique peut également consister à effectuer une démonstration directement devant vos interlocuteurs, d'un produit existant ou d'un prototype. Cela a beaucoup de valeur, mais nécessite d'être très soigneusement préparé.

Synthèse des techniques de représentation de scénarios d'usage

Parmi ces différentes techniques vous devrez choisir celle qui convient le mieux à votre projet, vos objectifs, aux observations réalisées ou aux utilisateurs que vous avez pu observer. Soyez créatifs !

La représentation de ces scénarios d'usage vous permettra alors d'avoir à disposition des synthèses pertinentes des problèmes et situations d'usage que vous allez analyser plus en détail dans la suite de votre projet.

\section{Notre conseil}

Choisissez une technique de représentation des usages adlaptée à vos ressources

Vous avez très probablement des ressources (temps, hommes, matériel) très limitées. Certaines techniques de représentation peuvent être très gourmandes en ressources, comme les films (temps de captation et de montage) ou les animations de type stop-motion (long post-traitement). Gardez bien en tête que ce n'est pas la qualité de la représentation qui est primordiale, mais le message qu'elle délivre. Par exemple, rien de sert de créer un film d'animation à 24 images par seconde pour mettre à jour des problèmes liés à la chute des personnes âgées ! Le message principal est donc de choisir à la fois une technique et un niveau d'ambition pertinents pour ce que vous souhaitez montrer.

\section{Évitez les erreurs}

Ne pensez pas que votre expérience peut remplacer celle des utilisateurs

Une solution de facilité souvent évoquée est d'observer ses propres usages ou ceux des membres de son groupe projet. C'est un début, qui peut vous orienter sur des observations plus ciblées, mais n'oubliez pas que votre expérience ne remplacera jamais celle de " vrais " utilisateurs, et que votre cas particulier n'est probablement pas valable pour tout le monde.

N'écoutez pas uniquement ce que l'utilisateur dit Les utilisateurs ont généralement beaucoup à vous apprendre. Pour autant, il y a également énormément de choses qu'ils ne savent pas exprimer, ou des besoins auxquels ils n'auraient même pas pensé. Pensez-vous que beaucoup d'utilisateurs auraient exprimé le besoin d'une tablette tactile quelques mois ou années avant que celles-ci n'apparaissent sur le marché ? Comment des personnes présentant un handicap mental peuvent-elles exprimer leurs besoins ? C'est à vous d'aller investiguer en profondeur pour découvrir ce qui n'est pas dit, ou ce qui est mal dit.

\section{Foire aux questions}

\section{Combien de scénarios d'usage représenter ?}

C'est une question qui est souvent posée dans le cadre de projets d'innovation Radical Innovation Design $®$. La réponse est bien sûr que cela dépend des ressources que vous avez à disposition, de ce que vous souhaitez faire de ces représentations, et du nombre d'usages que vous avez observés et qui méritent d'être relatés car étant tous très différents (contextes, usagers qui les vivent, histoires, problèmes et conséquences décrites...). Mais généralement, représenter de deux à cinq scénarios d'usage sur un problème bien ciblé vous donnera déjà un vocabulaire pour exprimer les principaux scénarios d'usage et problèmes à investiguer plus en détail, ce que nécessite la méthodologie RID. Vous devez chercher à couvrir le plus efficacement possible le périmètre de votre besoin idéal, c'est-à-dire couvrir au mieux l'ensemble des situations d'usage et de problèmes qu'il englobe.

\section{Existe-t-il des ressources gratuites pour réaliser} des scénarios d'usage?

Bien sûr. Ces quelques ressources gratuites, libres ou open-source peuvent vous aider à représenter des scénarios d'usage :

Pour les Story-boarding/bandes dessinées, vous pouvez vous aider de :

- Bitstrips

- Pixton

- Storyboardthat

- PowToon

En matière de films d'animation, vous pouvez consulter Synfig Studio.

\section{Pour aller + loin}

Sites internet

\section{Radical Innovation Design $®$}

Ce site propose de nombreux articles scientifiques. Ils vous permettront d'approfondir les différents aspects de la méthodologie Radical Innovation Design ${ }^{\circledR}$ (RID).

Site internet du cours Conception et Innovation de Produits et Services de l'Ecole Centrale Paris, utilisant la méthodologie RID

Retrouvez dans la section « Réalisation des étudiants » de nombreux exemples de résultats de projets RID, et notamment exemples de vidéos de scénarios d'usage existants.

Designing with people

Retrouvez sur ce site du Royal College of Art de Londres (ressource en anglais) de nombreuses techniques d'observation, expliquées dans des fiches synthétiques.

The methods lab - User research for design

Les auteurs du site "Designing with people » ont par ailleurs contribué à un guide très didactique présentant les méthodes d'observation, téléchargeable gratuitement (ressource en anglais). 


\section{Les plus Internet}

Saisissez la Référence Internet 1448 dans le moteur de recherche du site www.techniques-ingenieur.fr pour accéder aux mises à jour de cette fiche ainsi que la Réf. Internet des rubriques suivantes:

\section{Outils téléchargeables}

- dtou6473 - Modèle de fiche « Usage »

- dtou6474 - Exemple de fiche « Usage » : le plaquiste

\section{Fiches associées}

- 0424 - Réexprimer un besoin idéal à partir d'une idée initiale - La reproblématisation

- 0637 - Débuter un projet d'innovation - Le carnet de bord de l'investigation 


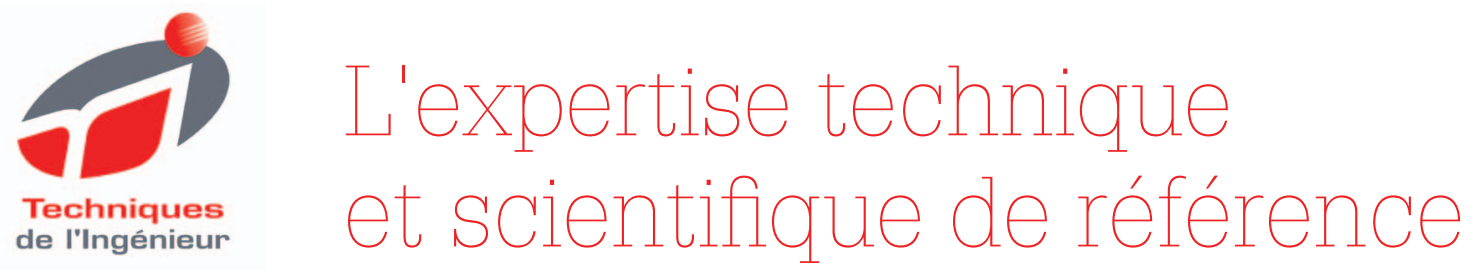

Techniques de l'Ingénieur vous apporte une information précise et fiable pour l'étude et la réalisation de vos projets. Actualisées en permanence, les ressources documentaires profitent aujourd'hui à plus de $\mathbf{3 0 0} \mathbf{0 0 0}$ utilisateurs sont la référence pour tout ingénieur, bureau d'études, direction technique et centre de documentation.

Depuis près de 70 ans, $\mathbf{3} \mathbf{5 0 0}$ experts contribuent quotidiennement à développer, enrichir et mettre à jour cette documentation professionnelle unique en son genre

L'intégralité de ces ressources représente plus de $\mathbf{9 0 0 0}$ articles, répartis dans plus de $\mathbf{4 3 0}$ bases documentaires, accessibles sur internet, en téléchargement PDF, et sur tablette.

4 BONNES RAISONS DE CHOISIR TECHNIQUES DE L'INGÉNIEUR

- Une actualisation permanente du fonds documentaire

- Un comité d'experts scientifiques et techniques reconnus

- Une collection scientifique et technique incontournable sur le marché francophone

- L'espace actualité pour suivre les tendances et innovations de vos secteurs

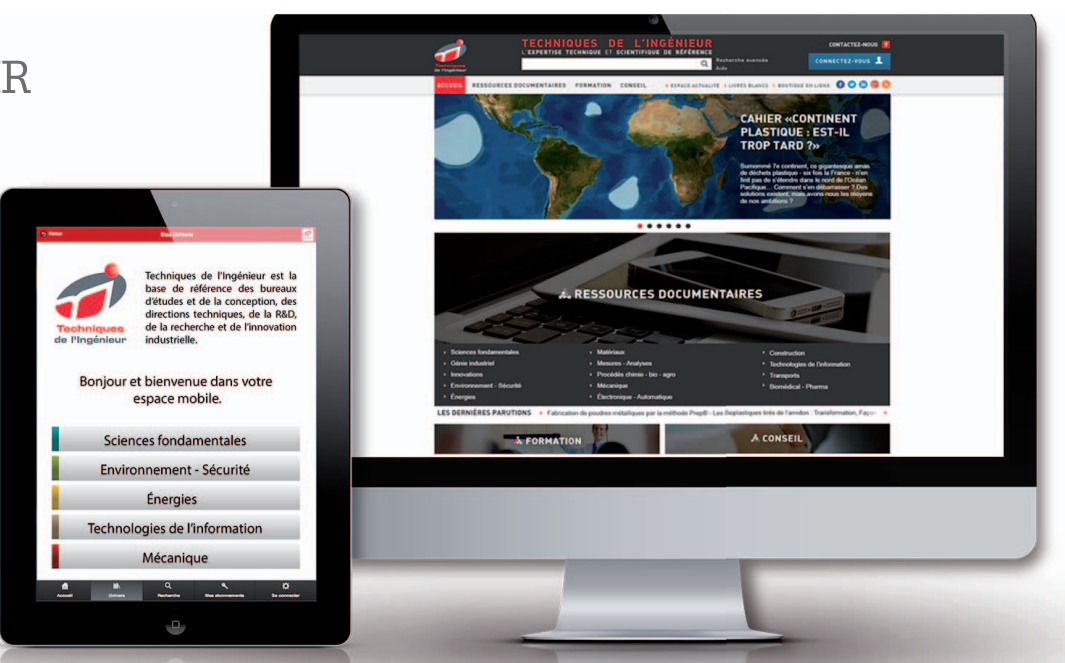

\section{DES SERVICES ASSOCIÉS À CHAQUE ABONNEMENT}

- Service de questions-réponses ${ }^{(1)(2)}$ : interrogez les plus grands spécialistes des domaines couverts par vos bases documentaires. Votre abonnement vous permet en effet de poser des questions techniques ou scientifiques.

- Les articles Découverte : un article vous intéresse, mais ne fait pas partie de votre abonnement ? Techniques de l'Ingénieur vous offre la possibilité de l'ajouter

- Le Dictionnaire technique multilingue : 45000 termes scientifiques et techniques - avec illustrations et légendes en français, anglais, espagnol, allemand.

- Les Archives : vos bases documentaires s'enrichissent et sont mises à jour en ligne en permanence. Les Archives conservent la mémoire de ces évolutions et vous permettent d'accéder aux versions antérieures de vos articles, ainsi qu'à ceux qui traitent des technologies plus anciennes

Profitez également de l'impression à la demande (1), pour commander une ou plusieurs éditions papier supplémentaires de vos bases documentaires (sur devis).

ILS NOUS FONT CONFIANCE :

ARKEMA COS DASSAULT
Sagemcom

L'ORÉAL

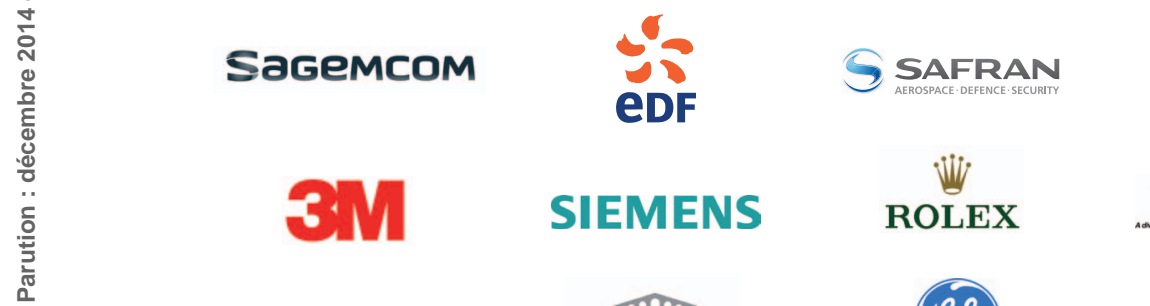

SIEMENS

$\operatorname{mim}_{1}$

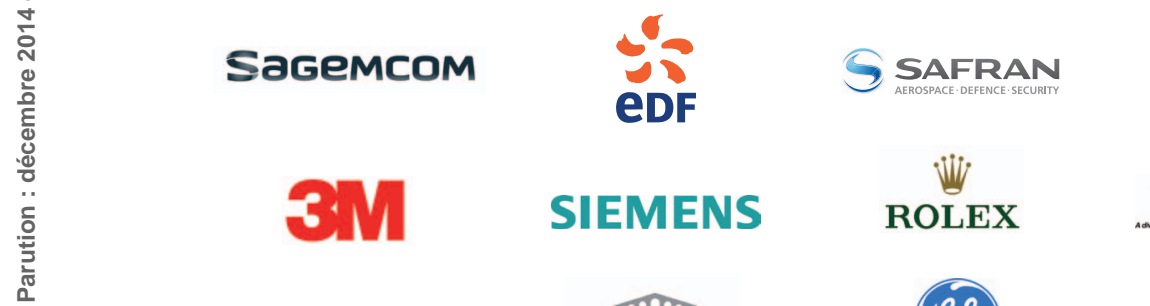

6 SAFRAN

iiii ROLEX

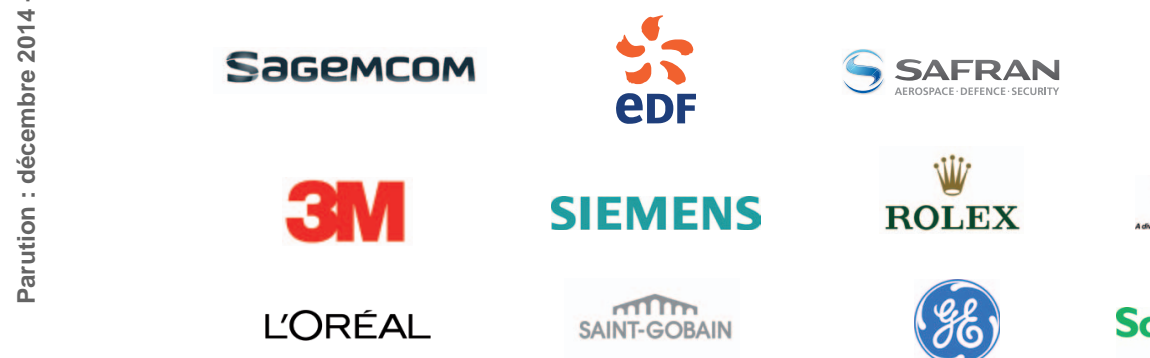




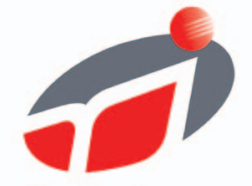

Techniques de l'Ingénieur
Pour disposer d'un panorama complet sur une thématique

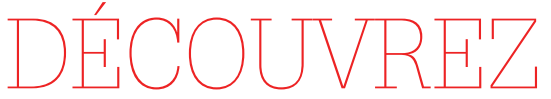

les offres de packs!

\section{LES + DES OFFRES PACK}

- Un large choix de + de 60 thématiques pour des besoins de contenu plus larges

- Des tarifs préférentiels sur mesure adaptés à vos besoins

LES UNIVERS DOCUMENTAIRES

- Plus de 430 bases documentaires et plus de 9000 articles en 14 univers
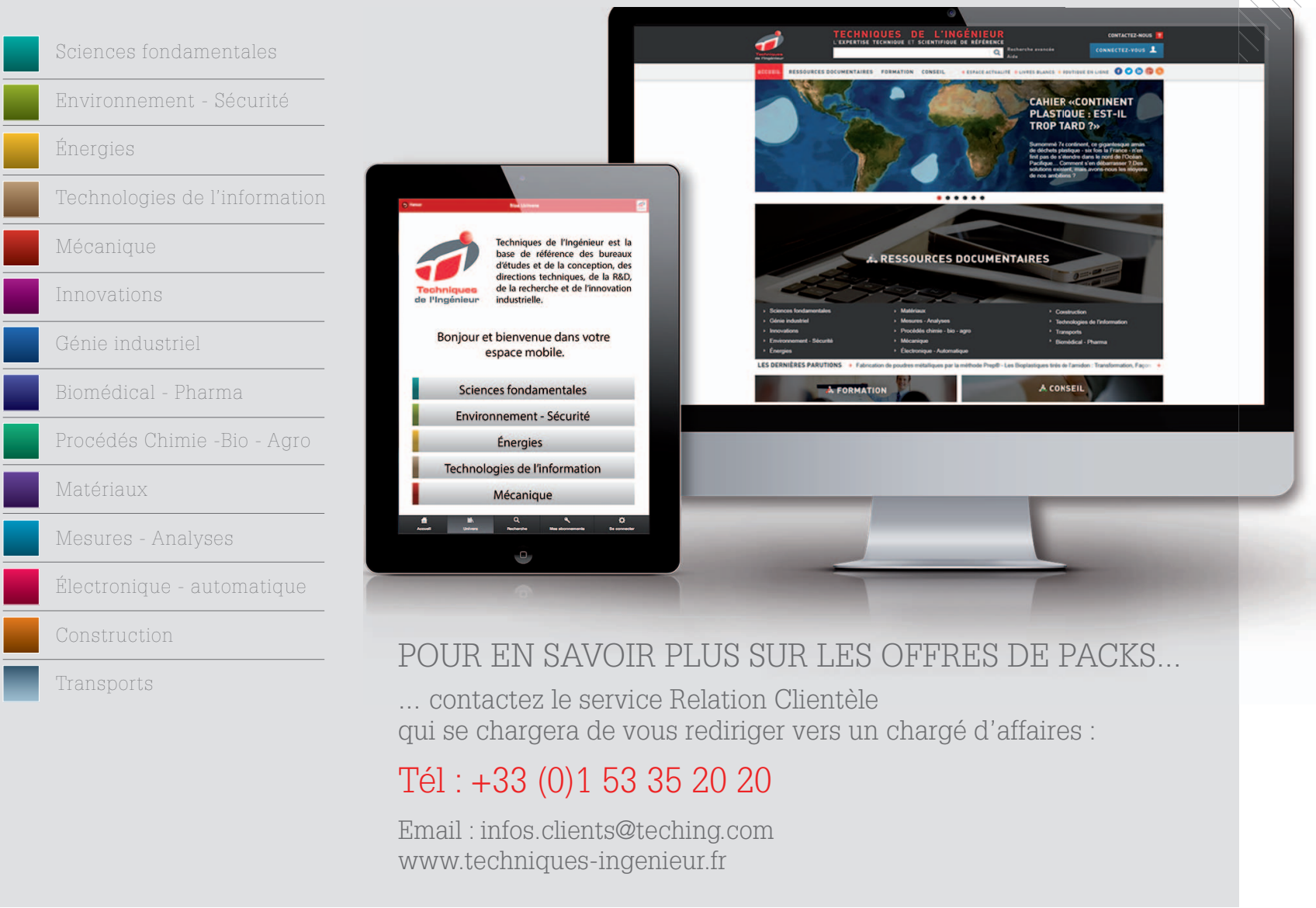

\section{LES AVANTAGES TECHNIQUES DE L'INGÉNIEUR}

ainsi que le téléchargement des versions PDF des articles de référence ou fiches pratiques inclus dans ces ressources. Les droits d'accès sont proposés en monoposte ou multiposte.

- ACTUALISATION PERMANENTE

Mises à jour permanentes, publication de nouveaux articles de références et fiches pratique : un contenu complet sur le sujet qui vous intéresse, des alertes par email.

- DES SERVICES INCLUS

En plus de l'accès aux ressources documentaires, chaque souscription offre un accès privilégié à un ensemble de services.

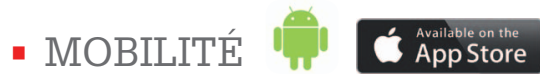




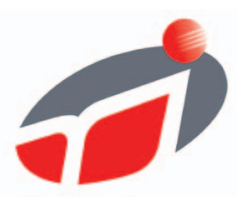

Techniques de l'Ingénieur

\section{MONTEZ EN COMPETENCE}

- Des formations personnalisées, réalisées au sein de votre établissement et à vos dates

- Un accompagnement à la mise en conformité réglementaire

- Des missions d'audit et de recommandations techniques

\section{LES ENGAGEMENTS TECHNIOUES DE L'INGÉNIEUR}

- Un réseau d'experts reconnus pour vous conseiller

- Une veille scientifique et technique pour mieux décider

- Les dernières obligations HSE pour être en règle

- Les clés en management des hommes et des projets pour gagner en efficacité

\section{Consultez l'intégralité}

des programmes sur le site

de Techniques de l'Ingénieur, espaces FORMATION et CONSEIL

www.techniques-ingenieur.fr

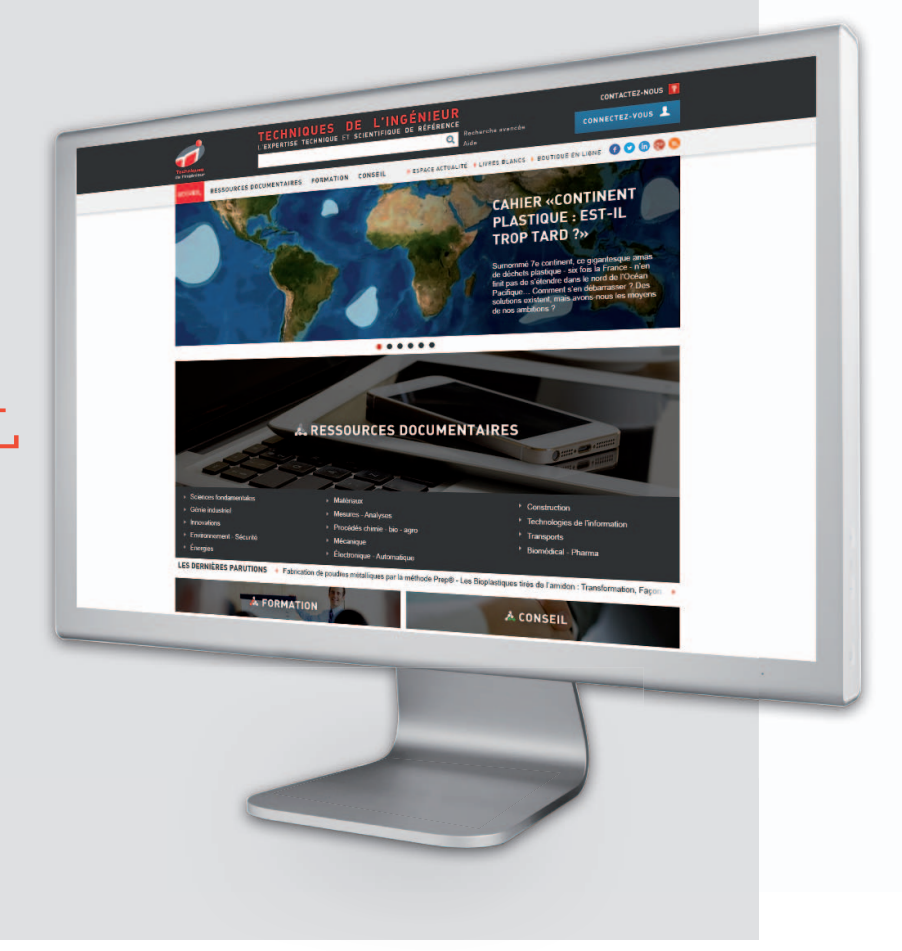

